\title{
The Good Wife : faits divers et fiction, allers-retours
}

The Good Wife: Crime News Items and Fiction, Back and Forth

\section{Anaïs Goudmand}

\section{OpenEdition}

Journals

Édition électronique

URL : http://journals.openedition.org/recherchestravaux/975

ISSN : 1969-6434

Éditeur

UGA Éditions/Université Grenoble Alpes

Édition imprimée

ISBN : 978-2-37747-056-3

ISSN : 0151-1874

\section{Référence électronique}

Anaïs Goudmand, «The Good Wife : faits divers et fiction, allers-retours », Recherches \& Travaux [En ligne], 92 | 2018, mis en ligne le 01 juin 2018, consulté le 08 septembre 2020. URL : http:// journals.openedition.org/recherchestravaux/975

Ce document a été généré automatiquement le 8 septembre 2020

(c) Recherches \& Travaux 


\title{
The Good Wife : faits divers et fiction, allers-retours
}

The Good Wife: Crime News Items and Fiction, Back and Forth

\author{
Anaïs Goudmand
}

1 Les séries qui s'inspirent de faits divers (« ripped-from-the-headlines», pour reprendre l'expression anglaise consacrée ${ }^{1}$ ) sont très nombreuses et se rattachent généralement à des genres spécifiques, notamment judiciaire (comme Law \& Order) ou policier (comme Criminal Minds). Au sein de cette riche tradition narrative, The Good Wife (2009-2016), série créée par Michelle et Robert King et produite par CBS, se distingue par la diversité de ses stratégies narratives et pose diverses questions sur la transposition du fait divers en fiction. La trame narrative globale des sept saisons est en effet très largement inspirée de faits divers notoires : l'héroöne, Alicia Florrick (Julianna Margulies), épouse du procureur général de Chicago, reprend sa carrière d'avocate lorsque son mari (Chris Noth) est accusé de corruption et impliqué dans un scandale sexuel. Pour le public américain comme pour le public français, l'allusion à l'« affaire Lewinsky ", qui a connu une très forte médiatisation dans le monde et qui a bouleversé l'image du couple Clinton, est évidente. De nombreux éléments de l'histoire d'Alicia Florrick sont également empruntés à un scandale plus récent qui a poussé le gouverneur de New York Eliot Spitzer à démissionner de son mandat en 2008, lorsque la presse a révélé ses relations avec des prostituées. En outre, au niveau des intrigues locales, les dossiers qu'Alicia prend en charge font souvent référence à des procès réels. La matière faitdiversière nourrit donc à la fois la dynamique sérielle et feuilletonnante de The Good Wife.

2 Cet aspect interroge le lien du fait divers avec le format narratif et la poétique des genres : le caractère éphémère du fait divers le cantonne-t-il dans la série télévisée à l'échelle de l'épisode? Dans quelle mesure peut-il s'incorporer aux intrigues à long terme du feuilleton, et plus particulièrement du feuilleton politique, qui présente des enjeux plus larges? Nous nous intéresserons également à l'insertion de la série dans l'espace médiatique et social, et plus précisément à l'inversion de l'effet de miroir entre faits divers et fiction. En effet, The Good Wife a confronté ses spectateurs à des expériences troublantes en mettant en scène des épisodes qu'on pourrait qualifier de 
"prémonitoires » dans la mesure où ils semblent anticiper des faits divers. Dans ce cas, l'effet de déjà-vu s'inverse, c'est la réalité qui semble transposer la fiction. Les phénomènes de collusion involontaire avec l'actualité s'avèrent particulièrement problématiques car ils échappent à l'intention des créateurs et résultent d'un effet de réception non planifié. À ce titre, ils se laissent a priori difficilement saisir dans le cadre d'une analyse stylistique. Pour autant, on peut se demander si ces effets défictionnalisants qui font de la fiction une grille de lecture du réel ne sont pas facilités par les stratégies narratives mises en œuvre par la série. En outre, nous étudierons la manière dont les diffuseurs se saisissent de ces coïncidences pour en faire des coups publicitaires en surenchérissant sur le sensationnalisme, quitte à orienter la réception vers d'autres possibles que ceux que les créateurs avaient imaginés.

\section{Du fait divers à la fiction : format narratif et poétique des genres}

3 The Good Wife se caractérise par sa mixité générique, puisque la série emprunte à la fois aux fictions judiciaires et politiques. Ces genres, du fait des éléments thématiques auxquels ils réfèrent, renvoient avant tout à la sémantique du récit, mais déterminent également l'organisation macrodiscursive de la série. Ainsi, tandis que la plupart des intrigues judiciaires s'inscrivent dans un format sériel, suivant un schéma éprouvé (un procès par épisode), les intrigues politiques fournissent quant à elles la matière de l'arc narratif surplombant.

4 En ce qui concerne tout d'abord l'intégration de faits divers au rythme épisodique de la série, The Good Wife reprend la formule qui a fait le succès de la série judiciaire Law and Order, entre autres : chaque épisode met en scène un procès différent qui fait souvent écho à l'actualité. Par exemple, l'épisode intitulé «Infamy» (saison 1, épisode 11, 2010) est similaire à un fait divers datant de $2006^{2}$ : il évoque le suicide d'une mère accusée par un commentateur de télévision d'avoir assassiné son enfant. La firme d'Alicia, Lockhart \& Gardner, représente le mari de la victime qui accuse la chaîne de télévision. De même, dans «Long Way Home » (saison 3, épisode 17, 2012), Lockhart \& Gardner prend en charge le dossier d'un PDG d'entreprise évincé qui cherche à retrouver son poste, mais qui est accusé de harcèlement sexuel par une ancienne employée, ce qui est très largement inspiré de l'affaire qui a coûté à Mark Hurd son poste de PDG de HewlettPackard (2010). Les exemples de ce type sont nombreux, car le fait divers repose à la fois sur une temporalité brève et sur une dimension sensationnelle qui le prêtent à une transposition épisodique. La dramatisation fait partie du contrat générique des séries judiciaires - ce qui vaut d'ailleurs également pour les autres genres qui se consacrent à un univers professionnel (policier, médical, politique...). Cette dramatisation tend à produire un récit évinçant les aspects les plus routiniers des affaires pour se concentrer sur les cas qui sortent de l'ordinaire afin de préserver ses qualités intrigantes, ce qui implique un ramassage de la temporalité des procès, expédiés en un épisode ${ }^{3}$. Cet aspect est parfaitement assumé par Michelle et Robert King, pour lesquels la dynamique narrative doit en l'occurrence primer sur la conformité avec la réalité du travail d'avocat: «La manipulation de la loi à des fins dramatiques a une longue et glorieuse histoire en ce qui concerne les séries juridiques ${ }^{4}$.» L'objet premier n'est donc pas d'offrir une image parfaitement réaliste du milieu, mais un récit passionnant, et le fait divers, en tant que " dérogation à une norme ${ }^{5}$ ", remplit parfaitement les critères 
de la narrativité et constitue ainsi une valeur sûre pour les créateurs. Nous ne nous attarderons cependant pas davantage sur ce traitement narratif du fait divers car il a été très largement exploité par de nombreux récits, télévisés aussi bien que littéraires, et renvoie ainsi à des régularités génériques bien identifiées.

Comme nous l'avons signalé, The Good Wife s'inspire d'affaires qui mettent en cause des représentants politiques. L'entremêlement des sphères privées et publiques qu'elles supposent entraîne des répercussions plus larges, qui débordent le cadre anecdotique. Le récit dépasse ainsi la circonscription dans le temps court du fait divers, pour l'inscrire dans une dynamique feuilletonnante et explorer ses conséquences à moyen et long terme sur la carrière d'Alicia. The Good Wife raconte ainsi la libération d'une épouse longtemps contrainte à réprimer ses ambitions professionnelles pour favoriser celles de son mari, en montrant la manière dont l'héroïne parvient progressivement à retourner une situation désavantageuse en sa faveur. Contrairement aux procès traités en un seul épisode, pour lesquels l'identification du fait divers prétexte importe finalement assez peu, la question de la mémoire et de la reconnaissance est ici primordiale. En effet, l'histoire d'Alicia ne prend sens que par les effets de symétrie qu'elle suscite avec l'actualité. La télévision (et Internet dans une part croissante) constituant le principal moyen d'information, le support langagier du fait divers n'est plus exclusivement textuel: les sources audiovisuelles constituent une source d'influence fondamentale pour la matière fictionnelle.

6 Les King ont ainsi choisi comme point de départ une image devenue iconique, celle de l'épouse bafouée qui soutient son mari dans les pires marasmes. Le principe initial consiste alors à interroger les non-dits derrière le vernis de solidarité conjugale :

Il y avait eu une cascade de ce type de scandales, de Bill et Hillary à Dick Morris et à Eliot Spitzer, pour n'en citer que quelques-uns. Je pense qu'ils envahissent notre culture. Et on retrouvait toujours l'image du mari en train de s'excuser avec sa femme à côté. Je crois que la série a débuté quand nous nous sommes demandé : «À quoi peuvent-elles bien penser ${ }^{6}$ ?»

7 La composition de la scène d'ouverture de la série, qui représente la démission de Peter Florrick, reprend très exactement de nombreux éléments visuels et discursifs de celle de Spitzer: présence de l'épouse apparemment stoïque à droite de son mari, phraséologie identique, fondée sur une rhétorique de l'excuse qui mobilise les mêmes collocations $^{7}$ (les isotopies de la repentance et de la famille notamment). La comparaison entre ces phrases, la première extraite du discours de démission de Spitzer, la seconde de celui de Peter Florrick, suffit à dévoiler les relations interdiscursives qui rapprochent les deux extraits :

Ces derniers jours, j'ai entrepris d'expier mes crimes personnels auprès de ma femme Silda, de mes enfants et de toute ma famille ${ }^{8}$.

Je dois expier mes fautes personnelles auprès de ma femme, Alicia, et de nos deux enfants'.

8 Le discours fictionnel procède donc par allusion en proposant une répétition avec variations du discours source. Ce phénomène correspond à la catégorie du sur-énoncé, telle que la définit Jousset: "La démarcation d'un patron, renouvelé par le détournement que l'allusion, en s'en prévalant ou en le subvertissant, lui fait subir ${ }^{10}$.» En entendant dans la bouche de Peter Florrick un discours qui semble familier et préfabriqué, les téléspectateurs sont ainsi amenés à remettre en cause sa sincérité : son repentir est sapé par le recours à des "éléments de langage ", suivant l'expression figée issue du domaine de la communication politique. 
9 Mais cette séquence ne repose pas uniquement sur un régime allusif, elle propose également des éléments saillants de reconfiguration fictionnelle. D'une part, elle adopte un point de vue subjectif ${ }^{11}$ en se concentrant immédiatement sur l'héroïne murée dans le silence et sur ses sensations. Pour reprendre la typologie de François Jost $^{12}$, le recours à l'ocularisation interne (observation des détails qui retiennent l'attention d'Alicia : gouttes de sueur sur le visage de son mari, fil blanc sur sa manche de costume...), à l'ocularisation modale (images mentales qui traversent l'esprit d'Alicia, imaginant Peter au lit avec une autre femme), et à l'auricularisation interne (le discours de Peter se brouille progressivement au fur et à mesure que l'héroïne se déconcentre, jusqu'à n'être plus qu'un bruit de fond) permet au spectateur de se situer au plus près des perceptions d'Alicia et de ressentir son malaise. D'autre part, la scène qui succède immédiatement se déroule en coulisses et donne à voir ce qui reste inaccessible dans le cas des couples Spitzer ou Clinton, à savoir la réaction de l'épouse lorsqu'elle n'est plus guettée par les caméras et lorsqu'elle peut sortir du rôle qui lui est assigné. En l'occurrence, Alicia gifle violemment son mari. Le récit fictionnel sert ici à interroger et à problématiser les lacunes de l'information initiale, à creuser et à complexifier les questionnements que peuvent susciter de tels scandales, tout en satisfaisant un appétit voyeuriste, puisque les caméras des réalisateurs peuvent prendre le relais là où celles des journalistes ne sont pas autorisées à s'aventurer. D'une certaine manière, la série commence là où le fait divers s'arrête. On peut supposer que la transposition fictionnelle de cette stratégie de communication bien rôdée (l'époux repentant, l'épouse silencieuse) a pu contribuer à enrayer son mécanisme de fonctionnement : elle met en exergue le caractère stéréotypique et construit de l'image récurrente, lui fait perdre sa crédibilité en exhibant son artificialité et son sous-texte idéologique, et remet ainsi en cause l'obligation tacite pour les épouses d'apparaître auprès de leur mari disgracié en défigeant ce qu'on pourrait appeler un interdiscours visuel $^{13}$.

Cette incidence de la fiction sur la perception d'images documentaires est en tout cas revendiquée par les créateurs :

À ce stade, dit Michelle King, les images sérielles de ces épouses exhibées au public à des moments aussi vulnérables ont commencé à apparaitre comme des clichés. Tout le monde est maintenant conscient des calculs politiques qu'elles impliquent, dit-il, et il se plaît à croire que la série a aidé à «mettre un terme » à de telles humiliations publiques ${ }^{14}$.

11 Pour analyser ce phénomène en termes narratologiques, la lecture sérielle d'images reliées entre elles par un « air de famille » permet d'isoler au sein de l'interdiscours une séquence narrative stéréotypique, le «scandale sexuel impliquant un homme politique", qui se conclut par un dénouement apparemment positif puisque le responsable politique défaillant est condamné. Mais des éléments dysphoriques rendent ce dénouement insatisfaisant, puisqu'un personnage secondaire sympathique est également puni : l'épouse, qui en plus d'être innocente, est la principale victime des écarts de son mari et se retrouve injustement sanctionnée par une humiliation publique. Des incertitudes laissées par cette séquence dérive une nouvelle séquence hétérogène, dans laquelle le protagoniste cède le devant de la scène narrative au personnage secondaire: The Good Wife débute ainsi par le dénouement d'une première séquence narrative implicite qui renvoie à un "scénario intertextuel ${ }^{15}$ " familier des spectateurs, et la mise en intrigue feuilletonnante s'élabore à partir de la construction d'un personnage qui s'émancipe du carcan narratif du fait divers initial. 
12 Le rythme narratif évolue en fonction du parcours professionnel de la protagoniste. Ainsi, on peut observer un changement notable à partir de la cinquième saison : lorsque l'héroïne se lance à son tour dans une carrière politique, les procès prennent une importance secondaire et le tropisme feuilletonnant est encore plus marqué. En effet, Alicia présente sa candidature au poste de Procureur de l'État d'Illinois, et n'apparaît plus que marginalement au barreau. La formule qui consiste à mettre en scène un procès par épisode est donc délaissée au profit d'une intrigue suivie tout au long de la saison, consacrée à sa campagne électorale. Toutefois, la série se risque ponctuellement à explorer des sujets d'actualité brûlante dans une limite épisodique. Ainsi l'épisode intitulé «The Debate » (épisode 12, saison 6), diffusé le 11 janvier 2015 : le débat entre Alicia et son concurrent est interrompu par le verdict du jury d'un procès contre deux policiers qui ont abattu un homme noir. L'épisode fait référence à l'affaire Eric Garner, tué le 17 juillet 2014 à Staten Island, ainsi qu'à l'affaire Michael Brown, qui a entraîné des émeutes à Ferguson en août 2014. Dans les deux cas, les policiers ont été innocentés par le jury (le 24 novembre 2014 en ce qui concerne la mort de Michael Brown et le 3 décembre 2014 dans le cas d'Eric Garner), ce qui a entraîné de nouvelles émeutes à Ferguson et des manifestations antiracistes dans l'ensemble du pays. Un texte d'avertissement apparaît au début de l'épisode :

Cet épisode a été écrit et filmé avant la décision du grand jury à Ferguson et Staten Island.

Toutes les mentions de «Ferguson » font référence aux événements d'août 2014, après la mort par balles de Michael Brown (n. t.).

13 Le texte introductif permet donc de récapituler deux informations extradiégétiques jugées nécessaires à la compréhension de l'épisode. D'une part, la très brève synthèse de la mort de Michael Brown éclaire des éléments de l'intrigue qui seraient restés inintelligibles pour des spectateurs auxquels l'affaire aurait échappé. En effet, le nouveau cas de violence policière dont il est question dans la série fait craindre aux personnages "un autre Ferguson", et tout l'enjeu est d'éviter la répétition d'événements dramatiques. D'autre part, le texte propose un éclaircissement concernant les connaissances dont disposaient les créateurs lorsqu'ils ont écrit le scénario: ils ont choisi d'évoquer une affaire en cours, et donc non résolue, le dénouement de l'épisode est à ce titre indépendant du procès réel. Mais la diffusion, quant à elle, a lieu après le verdict, les téléspectateurs se trouvant au moment du visionnage dans une position de supériorité cognitive par rapport aux scénaristes au moment de l'écriture. Il s'agissait d'un choix risqué de leur part dans la mesure où la mention du calendrier d'écriture ne les protège pas du parallèle avec les événements réels, qui font alors partie de l'« encyclopédie ${ }^{16}$ " partagée à laquelle se réfère le public pour interpréter l'épisode. La comparaison s'avère cruelle pour la série. En effet, dans l'épisode de The Good Wife, le jury conclut à l'innocence des policiers. Peter Florrick, désormais Gouverneur de l'Illinois, et l'épouse de la victime s'adressent aux manifestants et parviennent à éviter une émeute. À Ferguson, les appels au calme de Barack Obama et de la famille de Michael Brown sont en revanche restés lettre morte et n'ont pas permis d'éviter de nouveaux embrasements. «The Debate » s'est dès lors attiré de nombreuses critiques pour son dénouement irénique et sa focalisation sur les seuls personnages blancs, qui monopolisent la scène énonciative. Le journaliste David Sims le qualifie par exemple de "faible charge contre le racisme ${ }^{17}$ ", et la plupart de ses collègues vont dans le même sens : 
Malgré tous ses discours sur la politique progressiste et sa reconnaissance du racisme institutionnalisé, cet épisode de The Good Wife privilégie tout de même ses personnages blancs, leur attribue tous les moments d'émotion, qui sont ancrés dans leurs problèmes ${ }^{18}$.

Parler des éléments raciaux revient à ne parler de rien en particulier, parce que The Good Wife n'a pas de véritable intérêt pour eux ${ }^{19}$.

L'improvisation de la série sur ces situations est reléguée à l'intrigue $C$ de l'épisode, ce qui signifie qu'elle fonctionne essentiellement comme une distraction dans la vie des personnages principaux ${ }^{20}$. étrangère au format choisi pour traiter ce sujet. En cantonnant les affaires de violence policière à un épisode unique - et même à une sous-séquence adventice -, les scénaristes les ont réduites à des faits divers immanents ${ }^{21}$, qui ne questionnent pas sérieusement l'ordre établi : la paix des élites est préservée, notamment grâce à l'intervention du Gouverneur (blanc), le problème est considéré comme réglé et l'intrigue peut passer à autre chose. Parfois considéré comme le " pire épisode ${ }^{22}$ » d'une série par ailleurs jugée d'une excellente tenue, "The Debate » a fait office de révélateur des manques de The Good Wife sur la question de la représentation des minorités en illustrant les biais des médias dominants que dénoncent des mouvements comme " Black Lives Matter». Les militants afro-descendants s'insurgent en effet contre les tentatives de récupération politique auxquels ils sont constamment confrontés, et la manière dont la série traite le sujet est révélatrice de son aveuglement à cette question, puisqu'elle privilégie un point de vue centré sur les élites blanches persuadées qu'une réconciliation est possible. Ainsi, en dépit de son parti-pris engagé, The Good Wife échoue sur les questions raciales faute de leur accorder l'ampleur narrative qui lui a justement permis de développer ses problématiques féministes. Il est intéressant de noter que le choix de Robert et Michelle King de situer le spin-off de The Good Wife, The Good Fight, diffusé depuis février 2017, dans une agence qui emploie majoritairement des Noirs et défend entre autres les victimes noires de violences policières, résulte certainement d'une prise en compte de ces critiques.

Lasages du fait divers dans la The Good Wife sont donc variés. En premier lieu, de manière conforme au genre des séries juridiques, les faits divers nourrissent les procès qui fondent le rythme sériel du programme. Ensuite, la série se déploie en feuilleton en s'intéressant à une figure secondaire et sous-considérée des faits divers. Le parti-pris inverse, qui consiste à réduire la transposition fictionnelle de sujets d'actualité complexes à un seul épisode, est lourdement sanctionné par la critique: lorsque des faits divers sortent de leur rubrique pour devenir des faits de société cristallisant des conflits parfois violents, ils souffrent mal de redevenir simples faits « divers » dans la fiction. Dans tous les cas, il s'agit d'usages planifiés par la production (qui ne vont pas toujours sans heurts, comme nous avons pu le voir). Nous nous proposons de nous intéresser dans la partie suivante au mouvement inverse, lorsque les événements fictionnels de The Good Wife paraissent anticiper des événements réels.

\section{De la fiction au fait divers : effets de réception non planifiés}

Une fois que la série est bien installée dans le paysage culturel, on assiste à une inversion de l'effet de miroir : ce n'est plus le réel qui permet d'interpréter la fiction, 
mais la fiction qui devient une grille de lecture du réel. Ainsi, quand Eliot Spitzer tente de relancer sa carrière quatre ans après le scandale, il est scruté à travers le prisme de The Good Wife :

C'est quelque chose de particulier, quand l'art imite la vie, et qu'ensuite la vie se met à imiter l'art en retour. Mais c'est ce qui se passe dans le cas de la récente résurrection politique d'Eliot Spitzer et de sa relation avec son épouse Silda Wall Spitzer - ou de The Good Wife... dans la vie réelle, comme il faudrait désormais appeler cette histoire ${ }^{23}$.

Ce type d'expérience est troublant pour le public, mais d'autres cas s'avèrent plus déstabilisants encore. Dans «VIP Treatment " (saison 2, épisode 5, 2010), Alicia prend en charge le dossier d'une masseuse qui accuse un Prix Nobel de la paix de l'avoir agressée sexuellement dans une chambre d'hôtel. L'épisode est diffusé aux États-Unis quelques mois avant l'affaire du Sofitel qui a mis fin à la carrière politique de Dominique StraussKahn en mai 2011. Cette coïncidence est très largement dramatisée par M6, qui retransmet l'épisode en France en novembre 2011 et le présente selon ces termes dans la bande-annonce : "Cette enquête de The Good Wife a été diffusée en octobre 2010 aux États-Unis. » Le montage des extraits choisis et la voix off soulignent indirectement le parallèle avec le récent scandale: «Une femme seule... Un homme influent... Une affaire de mœurs... Deux vérités qui s'affrontent... Deux destins bouleversés. » Il n'est pas pour autant nécessaire aux diffuseurs de contextualiser davantage: l'affaire a connu une telle médiatisation qu'elle fait alors partie des références largement partagées des téléspectateurs français, il suffit donc de faire appel à leur mémoire immédiate et à leur capacité d'inférence - ce qui permet en outre de jouer sur l'oblitérabilité du sous-entendu (autrement dit, ce qui permet au locuteur de prétendre ne pas avoir voulu dire ce qu'il a dit ${ }^{24}$ ). La fameuse précaution rhétorique qui clôt la vidéo prend dès lors une saveur particulière: "Toute ressemblance avec des personnages existants ou ayant existé serait purement fortuite.»L'antériorité de l'écriture de l'épisode par rapport à l'affaire DSK vaut comme clause de nonresponsabilité pour M6, qui peut ainsi se réapproprier ironiquement la formule d'usage et verser dans le sensationnalisme sans pour autant être soupçonné de préjudice moral ${ }^{25}$. Dans les faits cependant, Michelle et Robert King se sont inspirés d'une autre affaire, avec laquelle le jeu de ressemblance s'avère encore plus frappant: Al Gore (luimême détenteur d'un Prix Nobel de la paix) a été accusé d'agression sexuelle par une masseuse en 2010. Ces effets de réception ne révèlent donc évidemment pas les capacités des scénaristes à prévoir l'avenir, mais bien plutôt la sérialisation médiatique des faits divers qui se succèdent, et partant l'identification de la séquence narrative stéréotypique relevée précédemment: rien ne ressemble plus à un scandale sexuel qu'un autre scandale sexuel. En les incorporant à sa matière narrative, la série se rend ainsi poreuse à l'opportunisme médiatique des diffuseurs, qui ne laissent jamais passer l'aubaine d'un coup publicitaire.

19 Analysons un dernier exemple intéressant. L'architexte du "fait divers », en tant que genre narratif plus ou moins stabilisé, est souvent traité avec distance par les auteurs. Laetitia Gonon relève ainsi un paradoxe qui préoccupait déjà les romanciers du XIX ${ }^{e}$ siècle :

Si [...] les romanciers s'inspirent effectivement du fait divers, ils le jugent aussi volontiers trop invraisemblable ou fictionnalisé, figé dans des topoï et des clichés, pour paraître utilisable en tant que tel - alors même qu'ils en font ensuite de la fiction. C'est donc désormais le récit non-fictif qui est trop excessif dans sa 
représentation du réel, et le récit fictif qui met en scène des événements ordinaires, réalistes - le roman doit être plus vrai que le journal : on voit bien là le paradoxe ${ }^{26}$. traduire par une stratégie inverse : plutôt que de recentrer le récit sur les éléments les plus crédibles des dossiers, les auteurs tendent alors à verser dans la caricature pour se démarquer des productions journalistiques. Prenons le cas d'un des personnages récurrents les plus emblématiques de The Good Wife, Colin Sweeney (Dylan Baker). Il s'agit d'un milliardaire flamboyant, doté d'un humour macabre, avec lequel Alicia entretient une relation ambiguë : elle ne peut s'empêcher de l'apprécier tout en étant persuadée qu'il est coupable du meurtre de sa femme, dont elle l'a pourtant innocenté. Robert et Michelle King n'ont pas revendiqué l'inspiration directe d'un fait divers en particulier pour Sweeney, qui apparaît plutôt comme une parodie des meurtriers hauts en couleur qui apparaissent dans les True Crimes $^{27}$ et leurs avatars et fait généralement office de contrepoint comique. Par ses traits de caractère outrés, son cynisme et son machiavélisme, il s'inscrit dans une architextualité immédiatement reconnaissable, qui ne peut être convoquée sans ironie. Les scénaristes le placent souvent dans des situations aussi loufoques que sordides. Par exemple, dans l'épisode "Hybristophilia " (saison 1, épisode 22, 2010), Alicia, venue chez lui pour lui faire signer un document, le retrouve menotté à un cadavre de femme, dont il prétend qu'elle a tenté de l'assassiner. Dylan Baker déclare à propos de son personnage :

L'équipe de scénaristes mérite vraiment son salaire quand il s'agit des épisodes de Colin Sweeney, parce qu'ils me mettent dans des situations très drôles, et je peux faire les choses les plus folles ${ }^{28}$.

21 Cet aspect est particulièrement frappant dans l'épisode intitulé « Dark Money » (saison 6, épisode 13), diffusé le $1^{\text {er }}$ mars 2015. Sweeney fait appel à la firme d'Alicia pour un procès en diffamation qu'il intente contre le créateur d'une série intitulée Call It Murder, dont un épisode représente un personnage qui lui ressemble fortement en train d'assassiner sa femme. Il s'agit évidemment d'une mise en abyme qui évoque le cassetête judiciaire que constituent les procès contre des représentations fictionnelles. La mise en scène du meurtre dans la série enchâssée pastiche très visiblement les séries « ripped-from-the-headlines » dont elle reprend, en les poussant à l'extrême, les motifs les plus identifiables: musique dramatique, éclairage blafard, rire démoniaque de l'assassin... C'est l'occasion d'un nouveau numéro de cabotinage pour Dylan Baker qui interprète à la fois Colin Sweeney, le meurtrier fictionnel nommé Bernard Loomis dans Call It Murder, dont quelques extraits sont diffusés lors du procès, et l'acteur anglais qui joue le rôle de Bernard Loomis. Le choix d'un acteur unique pour incarner les différents personnages accentue de manière comique la mauvaise foi du scénariste :

- Est-ce vous qui avez écrit cela, M. Tierney?

- C'est moi.

$-[. .$.$] Est-ce que c'est Colin Sweeney?$

- Non. Non, c'est un personnage fictif nommé Bernard Loomis.

- Vous êtes au courant que mon client a été innocenté du meurtre de sa femme?

- Je suis au courant, mais, à nouveau, ce personnage n'est pas basé sur lui.

- Je vois. Mais vous avez choisi un acteur qui ressemble exactement à Colin

Sweeney.

- C'est une coïncidence.

- [...] Hum, M. Tierney, l'Association des Critiques de Télévision a cité vos propos :

« Tous nos épisodes sont directement tirés de faits réels. » Donc, de quel fait réel a été tiré cet épisode? 
- C'était une exagération. Pas tous les épisodes. Ce n'était pas le cas de celui-ci (n.t.). bourdieusien du terme ${ }^{29}:$ en utilisant les compétences génériques des spectateurs pour instaurer une complicité avec eux, la série se positionne comme un récit réflexif, qui remet ironiquement en cause les codes du genre dont il dérive partiellement. Alors que l'équipe de Sweeney semblait bien partie pour gagner le procès, un retournement de situation change la donne: la défense finit par assumer la source d'inspiration mais récuse l'accusation pour diffamation en affirmant avoir réuni de nouveaux éléments qui prouvent que le milliardaire a bien tué sa femme, et le procès se retourne contre lui. Parallèlement, la mini-série documentaire d'Andrew Jarecki pour HBO, The Jinx : The Life and Deaths of Robert Durst, est diffusée entre février et mars 2015. Jarecki avait précédemment réalisé un film de fiction, All Good Things (2010), inspiré d'une biographie du milliardaire Robert Durst. Accusé de trois meurtres, dont celui de sa femme en 1982, ce dernier n'a jamais été condamné faute de preuves concluantes. Après avoir vu All Good Things, il contacte Jarecki et lui propose des entretiens et un libre accès à ses archives, ce qui aboutit au documentaire. The Jinx fait grand bruit grâce à son dénouement spectaculaire : dans la séquence finale du dernier épisode, Robert Durst se rend aux toilettes et, oubliant la présence de son micro, fait un aveu involontaire : « Ce que j'ai fait? Je les ai tous tués, évidemment ${ }^{30}$. »Ce nouvel élément à charge conduit à son arrestation le 14 mars, veille de la diffusion du dernier épisode sur HBO. La proximité des dates de diffusion de The Jinx et de "Dark Money» rend la ressemblance entre Sweeney et Durst particulièrement frappante. Dans les deux cas, un milliardaire cynique innocenté du meurtre de sa femme est mis en danger par son hybris. Là encore, l'effet de réception est dû à une coïncidence de calendrier et non à une stratégie narrative planifiée, ainsi que le soulignent les créateurs :

H. Goldblatt : Est-ce que vous avez pensé à un cas du type Durst?

M. King: C'est-à-dire, vous savez, nous avons notre propre sociopathe à domicile,

Colin Sweeney. Je veux croire qu'on y a pensé avant HBO.

H. Goldblatt : C'est juste!

R. King: On va faire un procès, je veux dire, il est tout simplement en train de plagier le charme de Sweeney, c'est terrible! Mais c'est intéressant, c'est une version un peu plus ennuyeuse, Sweeney n'est pas du tout ennuyeux. [...] Mais ils ont eu de meilleurs «twists » dans la réalité, c'est fou à quel point les réalisateurs peuvent être chanceux parfois ! [...] Ce murmure à la fin... [...] On n'aurait pas osé écrire ça dans la salle de scénario ${ }^{31}$.

Ainsi, alors même que Sweeney est conçu comme un personnage rocambolesque, alors que de nombreux éléments de "Dark Money» pastichent ouvertement les séries «inspirées de faits réels ", les scénaristes soulignent qu'un tel dénouement aurait paru invraisemblable dans une fiction et s'avèrent battus à plate couture par le «twist » qui conclut The Jinx. Dans le cas du documentaire, le trouble vient du fait qu'il n'y a pas d'intentionnalité auctoriale à laquelle rapporter le retournement de situation qui apparaît, en dépit de ses incontestables qualités narratives, comme le fruit d'un hasard et donne l'impression d'une intrigue «naturelle ", issue du simple enregistrement d'un événement brut et indépendante du langage qui informe la mise en récit. Le malaise de Durst a effectivement été provoqué par Jarecki et son équipe, mais ceux-ci n'imaginaient pas un seul instant qu'il serait capable d'une telle imprudence. La scène finale de The Jinx ne constitue d'ailleurs pas le dénouement de l'affaire Durst: elle engendre une nouvelle incertitude (Durst sera-t-il finalement condamné ?) qui laisse 
l'histoire inachevée. Le documentaire n'est donc plus le récit rétrospectif d'une enquête, ainsi que le concevait initialement Jarecki, mais il se trouve pris dans un récit qui le déborde, et dont la suite est à retrouver dans les médias d'information. Dans la fiction au contraire, les auteurs sont toujours susceptibles d'être sommés de rendre compte de leurs décisions par le public, ce qui les incite à la prudence : les limites du vraisemblable que pose la création fictionnelle sont parfois dépassées par la réalité.

The Good Wife exploite ainsi de diverses manières les liens privilégiés qui unissent le récit de fiction et le fait divers, mais la plus spectaculaire est certainement celle qui échappe à l'intention des créateurs. Ce phénomène montre bien qu'autant que de décisions auctoriales, la sérialisation, au sens large du terme, et non exclusivement narratif, résulte du pôle de réception. Ainsi que le rappelle Guillaume Soulez :

Pensée depuis la réception plutôt que depuis la production («industrielle» ou industrialisée), la mise en série, au sens structurel, est la recherche d'une cohérence entre des objets partageant un air de famille, comme lorsqu'on parle de "série noire " pour évoquer une suite rapprochée de catastrophes naturelles ou aériennes, sans rapport a priori les unes avec les autres (par leur éloignement géographique en particulier). Le recours à des genres identifiés (western, polar...) ainsi qu'à des signatures ou à des comédiens connus, etc. permet une sérialisation de la culture qui s'offre aux lecteurs et leur permet de construire des repères pour situer les objets. Standards (genres, styles filmiques, professionnels dédiés à une convention particulière...) et marques (noms et signatures multiples, de l'écrivain au studio en passant par la star...) délimitent en particulier le champ culturel pour un public alphabétisé et consommateur d'une culture médiatique ${ }^{32}$.

De ce point de vue, The Good Wife prend sa place au sein d'une « encyclopédie » du fait divers qui mêle récits factuels et récits fictionnels, et fait partie du réseau de connaissances potentiellement mobilisable dans l'interprétation des événements réels auxquels les ressources médiatiques donnent accès. Il ne s'agit pas pour autant de verser ici dans le panfictionnalisme: la fascination que provoque l'inversion de l'effet de « déjà-vu » vient justement d'une compétence partagée, celle qui consiste à savoir distinguer fait et fiction.

\section{NOTES}

1. L'expression est souvent utilisée pour faire la promotion des épisodes ou dans les articles consacrés aux séries. Cependant, ainsi que le précise le site Wikia consacré à Law \& Order, le récit lui-même tend plutôt à insister sur la nature fictionnelle des événements racontés: "Les publicités des épisodes qui se rapprochent d'affaires réelles utilisent souvent la phrase "ripped from the headlines", bien que dans l'épisode lui-même, un avertissement textuel insiste sur le fait que l'histoire et les personnages sont fictionnels. Ce format se prête à l'exploration de conséquences ou de mobiles différents qui auraient pu être occasionnés par des événements similaires dans d'autres circonstances. » Notre traduction (n.t.) : "Promotional advertisements of episodes with close real-life case parallels often use the "ripped from the headlines" phrase, although a textual disclaimer, within the actual episode, emphasizes that the story and characters are fictional. This 
format lends itself to exploring different outcomes or motives that similar events could have had under other circumstances. ", <http://lawandorder.wikia.com/wiki/Ripped_from_the_headlines>.

2. Sur le suicide de Melinda Duckett suite à son interview par la présentatrice Nancy Grace pour $\mathrm{CNN}$, et sur le procès intenté par la famille de la jeune femme, voir notamment: <http:// edition.cnn.com/2006/LAW/11/21/nancygrace.sued/index.html ?eref =yahoo>

3. Raphaël Baroni rappelle ainsi le lien entre mise en intrigue et transgression de la routine : «Les actions passionnantes, singulières, polémiques ou planifiées, qui constituent l'armature principale des récits, s'opposent en effet à celles ennuyeuses, répétitives, coopératives ou automatiques, dont rendent compte les scripts dans la vie quotidienne » (R. Baroni, La Tension narrative. Suspense, curiosité et surprise, Paris, Seuil, coll. « Poétique », 2007, p. 175).

4. M. King, "The Good Wife: Non-Lawyers Behind That Lawyer Show ", Bitter Empire, 2010, n.t., <http://bitterempire.com/the-good-wife-non-lawyers-behind-that-lawyer-show/>.

5. G. Auclair, Le Mana quotidien. Structures et fonctions de la chronique des faits divers, Paris, éditions Anthropos, 1970, p. 35.

6. M. King, art. cité, n. t.

7. Le terme « collocation » désigne des cooccurrences privilégiées au sein d'un groupe de mots : ici, « expier mes crimes / fautes personnel(le)s », par exemple.

8. N. t. : «In the past few days, I've begun to atone for my private felons with my wife Silda, my children, and my entire family. "

9. N. t. : «... I need to atone for my personal failings with my wife, Alicia, and our two children. » Il est intéressant de remarquer comment les scénaristes de The Good Wife ont procédé par synonymie : la structure syntaxique est la même, mais l'adjectif private devient personal, et felons est remplacé par failings.

10. Ph. Jousset, « À propos des sur-énoncés », dans B. Buffard-Moret (dir.), Bons mots, jeux de mots, jeux sur les mots : de la création à la réception, Arras, Artois Presses Université, 2015, p. 13.

11. Rappelons que Dorrit Cohn envisageait la subjectivité comme l'un des principaux marqueurs de fictionnalité (Le Propre de la fiction [1999], Paris, Seuil, « Poétique » 2001). Si ce point de vue a été très largement contesté par les tenants d'une approche pragmatique, qui montrent qu'en vertu de la nature mimétique du discours fictionnel, il est impossible de délimiter le domaine de la fiction suivant des frontières internes aux textes (voir notamment J.-M. Schaeffer, Pourquoi la fiction ?, Paris, Seuil, « Poétique », 1999), il n'en reste pas moins que les récits fictionnels, tous supports médiatiques confondus, recourent plus systématiquement au point de vue subjectif que les récits factuels.

12. F. Jost, L'Cil-caméra. Entre film et roman, Lyon, Presses universitaires de Lyon, 1987.

13. Selon Dominique Maingueneau, le processus de reconfiguration est au cœur de l'interdiscours: "L'interdiscours consiste en un processus de reconfiguration incessante dans lequel une formation discursive est conduite [...] à incorporer des éléments préconstruits produits à l'extérieur d'elle-même, à en produire la redéfinition et le retournement, à susciter également le rappel de ses propres éléments, à en organiser la répétition, mais aussi à en provoquer éventuellement l'effacement, l'oubli ou même la dénégation » (Nouvelles tendances en analyse du discours, Paris, Hachette, 1987, p. 83).

14. K. Q. Seelye, «A New Twist to Wives' Playbook for Sex Scandals », The New York Times, 2011, n. t., <http://www.nytimes.com/2011/06/19/us/19wives.html>.

15. U. Eco, Lector in fabula, Paris, Grasset, 1985, p. 104.

16. U. Eco, ouvr. cité.

17. D. Sims, «"The Good Wife" Takes a Weak Swipe at Racism », The Atlantic, 2015, n.t., <https:// www.theatlantic.com/entertainment/archive/2015/01/the-good-wife-stumbles-trying-toconfront-institutional-racism/384448/>.

18. K. Kumari Upadhyaya, "The Good Wife: "The Debate" ", AVClub, 2015, n.t., <http:// www.avclub.com/tvclub/good-wife-debate-213704>. 
19. L. Hill, «"The Good Wife" Season 6 Episode 12 Recap: "The Debate" ", Flavorwire, 2015, n. t. : « To speak about the racial elements is to speak about nothing in particular because The Good Wife has no real interest in them. » <http://flavorwire.com/498311/the-good-wife-season-6-episode-12-recapthe-debate>.

20. T. VanDerVerff, "The Good Wife dealt with the Ferguson protests in its worst episode in years", Vox, 2015, n. t., < http://www.vox.com/2015/1/12/7530625/good-wife-recap-debate>.

21. L'immanence est l'une des principales caractéristiques de la structure du fait divers selon Barthes (R. Barthes, "Structure du fait divers ", Essais Critiques, Paris, Seuil, 1964, p. 188-198). Cependant, un grand nombre de faits divers de The Good Wife échappent à cette définition puisqu'ils dépassent la sphère du pur événement et touchent aux domaines politiques et médiatiques.

22. T. VanDerVerff, art. cité.

23. K. Fallon, «Eliot Spitzer's Comeback: "The Good Wife" in Real Life », The Daily Beast, 2013, n. t.

24. Voir O. Ducrot, Le Dire et le dit, Paris, Éditions de Minuit, 1984.

25. Le problème s'est posé en d'autres termes pour TF1 concernant la diffusion d'un épisode de New York, Unité Spéciale, quant à lui directement inspiré de l'affaire : après des hésitations, la chaîne finit par le diffuser selon le calendrier prévu, en juillet 2012, mais sans faire de coup publicitaire pour éviter la polémique.

26. L. Gonon, Le Fait divers criminel dans la presse quotidienne française du XIXe siècle, Paris, Presses Sorbonne Nouvelle, 2012, p. 229.

27. Le True Crime est un genre littéraire, filmique ou journalistique dans lequel sont relatés des crimes réels.

28. D. Baker, « Exclusive: Interview with Dylan Baker: Trick ' $r$ Treat and Spider-Man ", Nerd Reactor, 2013, n.t., <http://nerdreactor.com/2013/10/25/interview-dylan-baker-trick-r-treat-spider$\operatorname{man} />$

29. P. Bourdieu, La distinction. Critique sociale du jugement, Paris, Éditions de Minuit, 1979.

30. N. t. : "What the hell did I do? Killed them all, of course. »

31. M. et R. King, entretien avec H. Goldblatt, «The Good Wife creators on Kalinda leaving and a Robert Durst storyline », Entertainment Weekly Radio, 20 mars 2015, n. t.

32. G. Soulez, «La double répétition. Structure et matrice des séries télévisées », Mises au Point, 3/2011, « Sérialité : densités et singularités », 2011, § 27.

\section{RÉSUMÉS}

Cet article vise à proposer une approche narratologique et stylistique de la transposition du fait divers en fiction dans la série télévisée The Good Wife (CBS, 2009-2016). Il s'agit d'étudier d'une part la manière dont le fait divers s'articule à la mise en intrigue sérielle et feuilletonnante, d'autre part les effets de réception non planifiés, lorsque certains épisodes semblent anticiper des faits divers ultérieurs au processus de rédaction.

This paper aims at offering a narratologic and stylistic approach of the transposition of stories from the headlines to fiction in the TV show The Good Wife (CBS, 2009-2016). Firstly, it studies how these stories are hinged on episodic and serial emplotment. Secondly, it analyzes unplanned reception effects, when some episodes seem to anticipate stories that happen after the writing process. 


\section{AUTEUR}

\section{ANAÏS GOUDMAND}

Université de Lausanne (Suisse), CRAL (UMR 8566 CNRS/EHESS)

Agrégée de lettres modernes, ancienne élève de l'École normale supérieure de Paris, Anaïs Goudmand est assistante à l'université de Lausanne et doctorante au Centre de recherche sur les arts et le langage (CRAL) de l'EHESS sous la direction de Jean-Marie Schaeffer et à l'université de Lausanne, en Suisse, sous la direction de Raphaël Baroni (intitulé du sujet : « Sérialité et fictionnalité : pour une poétique du récit sériel »). Elle a récemment publié plusieurs articles sur la sérialité narrative : «Le roman-feuilleton ou l'écriture mercenaire : l'exemple des Mystères de Paris ", dans Alessandro Leiduan (dir.), Sérialité narrative. Enjeux esthétiques et économiques, Cahiers de narratologie, décembre 2016 ; “ “Oh my God! They've killed...!” Le récit sériel entre autonomie et hétéronomie : conséquences du départ non planifié des acteurs sur la production et la réception des séries télévisées ", dans Raphaël Baroni et François Jost (dir.), Télévision, nº 7, « Repenser le récit avec les séries télévisées ", mars 2016 ; ou encore « Les séries transmédiatiques : des univers sans fin ? ", dans Proteus - Cahiers des théories de l'art, n 9, « Fin de série », Simon Lefebvre \& Gary Dejean (dir.), septembre 2015. 